\title{
Angioprevention is I mplicated in Resveratrol Chemoprevention of Experimental Hepatocarcinogenesis
}

\author{
Anupam Bishayee*, Danielle M. Petit and Karishma Samtani
}

Cancer Therapeutics and Chemoprevention Group, Department of Pharmaceutical Sciences, Northeastern Ohio Universities Colleges of Medicine and Pharmacy, Rootstown, $\mathrm{OH} 44272$, USA

\begin{abstract}
Hepatocellular carcinoma ( $\mathrm{HCC}$ ), a hypervascular tumor, is one of the most common and lethal cancers worldwide. We previously showed that resveratrol, a dietary polyphenol, inhibits rat liver carcinogenesis through antioxidative and antiinflammatory mechanisms. As resveratrol possesses antiangiogenic properties, we hypothesize that it may exert chemoprevention of hepatocarcinogenesis by suppressing angiogenesis. The antiangiogenic effect of resveratrol was investigated by analyzing livers from our previous study in which resveratrol $(50-300 \mathrm{mg} / \mathrm{kg}$ ) exerted chemopreventive action against diethylnitrosamine (DENA)-induced rat liver tumorigenesis. Hepatic angiogenesis was evaluated by microvessel density (MVD) based on immunohistochemical staining of CD31-positive endothelial cells. The expression of hepatic vascular endothelial growth factor (VEGF) and hypoxia-inducible factor-1 $\alpha$ (HIF-1 $\alpha$ ) were determined by immunohistochemistry. Sixteen weeks following the administration of DENA, there was a substantial increase in hepatic MVD as compared to normal liver. A dramatic increase in hepatic VEGF and HIF-1 $\alpha$ was observed in DENA-treated animals compared to normal counterparts. Treatment with resveratrol dose-dependently abrogated the DENA-induced increased MVD as well as the elevated expression of VEGF and HIF-1 $\alpha$. DENA-initiated hepatocarcinogenesis in rats exhibits substantial neovascularization possibly due to overexpression of VEGF upregulated by HIF-1a. Resveratrol exerts a remarkable angiosuppressive effect in DENA-evoked hepatocellular carcinogenesis. Resveratrol-mediated inhibition of angiogenesis could be achieved by suppressing VEGF expression through downregulation of HIF1a. These results, in conjunction with our previous findings, provide evidence that angiosuppression is involved in resveratrol-mediated chemoprevention of rat liver carcinogenesis and support the potential use of this natural agent in the prevention and therapy of HCC.
\end{abstract}

Keywords: Angiogenesis; Angioprevention; Diethylnitrosamine; Hepatocarcinogenesis; HIF-1 $\alpha$; Liver MVD; VEGF; Resveratrol

\section{Introduction}

Primary liver cancer, mostly hepatocellular carcinoma (HCC), is a global health problem, and represents the fifth most common malignancy and the third most common cause of death worldwide [1]. Although more than $80 \%$ of newly diagnosed cases occur in Eastern Asia as well as sub-Saharan Africa, the incidence of HCC has been increasing steadily in Western countries [2]. According to the American Cancer Society [3], more than 24,000 new cases and nearly 19,000 deaths are estimated to occur in the United States due to liver cancer (including biliary cancers) in 2010 alone, ranking it as the third leading cause of death in men between the ages of 40 and 59. The majority of HCC patients have underlying chronic liver diseases, the etiology of which includes viral infection (hepatitis B and $\mathrm{C}$ ), alcohol abuse, metabolic disorders, and exposure to dietary hepatocarcinogens including aflatoxins and nitrosamines [4-7]. Surgical resection and liver transplantation are considered to be the curative treatments for HCC; however, only limited numbers of patients with localized tumors are amenable to these curative approaches [8]. The recent advent of non-surgical and locoregional therapies, such as ethanol injection, radiofrequency ablation and radioembolization, can improve the survival of selected patients with tumors confined to the liver but further add to the complexity of HCC management [9]. Sorafenib, the only drug approved by the United States Food and Drug Administration for the treatment of advanced HCC, increases the median survival time by less than three months [10]. However, this drug does not defer the symptomatic progression of the disease, costs about $\$ 5,400$ per month for treatment [11], and possesses severe adverse effects including a significant risk of bleeding [12]. In view of the severity of the disease and the limited treatment options, a critical need exists for novel chemopreventive strategies which reduce the current morbidity and mortality associated with HCC.

HCC represents one of the most vascular tumors and is associated with a high propensity for vascular invasion [13,14]. Angiogenesis, a process that leads to the formation of new blood vessels from preexisting ones, has been shown to play an important role in the growth and progression of HCC. The proliferation and migration of endothelial cells, constituents of blood vessels, result in the formation of microvessels as well as vascular networks. It has been well established that tumor angiogenesis is a complex multistep process driven by an imbalance of the downregulation of antiangiogenic factors and upregulation of proangiogenic factors [15]. Vascular endothelial growth factor (VEGF), a heparin-binding homodimeric glycoprotein, is the most important angiogenic factor that stimulates the growth and migration of the endothelial cells [16]. Expression of VEGF is regulated by microenvironmental as well as genetic alterations in tumor cells. Hypoxia due to elevated metabolic activities and oxygen consumption by rapidly proliferating tumor cells is considered to be a key microenvironmental factor of angiogenesis, and hypoxia-induced factors (HIFs) are known to stimulate VEGF [17]. Emerging studies provide convincing evidence that angiogenesis

*Corresponding author: Anupam Bishayee, M.Pharm., Ph.D., Department of Pharmaceutical Sciences, Northeastern Ohio Universities Colleges of Medicine and Pharmacy, 4209 State Route 44, Rootstown, OH 44272, USA, Tel: +330 325 6449; Fax: +330 325 5936; E-mail: abishayee@neoucom.edu

Accepted September 28, 2010; Published September 28, 2010

Citation: Bishayee A, Petit DM, Samtani K (2010) Angioprevention is Implicated in Resveratrol Chemoprevention of Experimental Hepatocarcinogenesis. J Carcinogene Mutagene 1:102. doi:10.4172/2157-2518.1000102

Copyright: (c) 2010 Bishayee A, et al. This is an open-access article distributed under the terms of the Creative Commons Attribution License, which permits unrestricted use, distribution, and reproduction in any medium, provided the original author and source are credited. 
is involved in the early stages of multistage hepatocarcinogenesis $[18,19]$. Hepatocellular carcinogenesis depends on stepwise progression from the preneoplastic lesions with inflammation to the neoplastic stages with initiation and promotion of angiogenesis. Consequently, interference of the angiogenic process could be a valuable strategy in the prevention of HCC. It has been postulated that the best approach to inhibit angiogenesis is to intervene as early as possible during the carcinogenic process, even prior to overt tumor formation. The link between angiogenesis and cancer development and the ease of detection of this process in accessible tissues early in carcinogenesis make angiogenesis as a biomarker and target in cancer chemoprevention [20]. It has been hypothesized that effective chemopreventive agents block tumor angiogenesis, a concept termed as "angioprevention" [21]. Indeed, several phytochemicals, including dietary agents belonging to diverse chemical groups with cancer chemopreventive activities, have been shown to share antiangiogenic properties [22-25]. Nevertheless, chemoprevention of HCC through attenuation of angiogenesis has not been investigated in detail.

Resveratrol (3,4',5-trihydroxy-trans-stilbene, Figure 1), a naturally occurring polyphenol present in grapes, red wine and peanuts, has been shown to possess potent anticancer effects against various human cancer cells in vitro and a chemopreventive effect against breast, colon, esophagus, lung and skin carcinogenesis in vivo [26,27]. Nevertheless, the mechanism-based chemopreventive action of this dietary polyphenol against HCC has not been investigated until recently [28]. Our laboratory has reported, for the first time, that dietary resveratrol affords chemoprevention of hepatic tumorigenesis in a two-stage model of hepatocarcinogenesis in rats initiated with the dietary carcinogen diethylnitrosamine (DENA) and promoted by phenobarbital (PB) [29]. Although the molecular mechanisms of liver tumor inhibitory effects of resveratrol have not been completely elucidated, we have found that resveratrol suppressed DENA-induced elevated levels of hepatic cell proliferation, inflammatory response, and oxidative stress and also triggered apoptosis of DENA-initiated hepatocytes in a dose-responsive fashion [29-32]. As abnormal cell proliferation, inflammation and reactive oxygen species play vital roles in angiogenesis [33,34], the antiproliferative, antiinflammatory and antioxidant agent resveratrol may have an antiangiogenic role, which may be linked to its previously observed antihepatocarcinogenic effect. In fact, recent studies have shown that resveratrol exhibits a potent inhibitory effect on tumor angiogenesis in vivo in chemicallyinduced skin carcinogenesis [35] as well as xenografted skin, breast, colon, pancreas, lung and brain cancer models in rodents [36-43]. The angiosuppressive effects of resveratrol could be mediated through the inhibition of HIF- $1 \alpha$ and VEGF expression as observed in various cancer cell lines [44-48]. In view of these studies, we hypothesize that resveratrol-mediated chemoprevention of experimental hepatocarcinogenesis could be achieved, at least in part, through suppression of neovascularization. The objective of the present study was to investigate the effects of resveratrol on angiogenesis

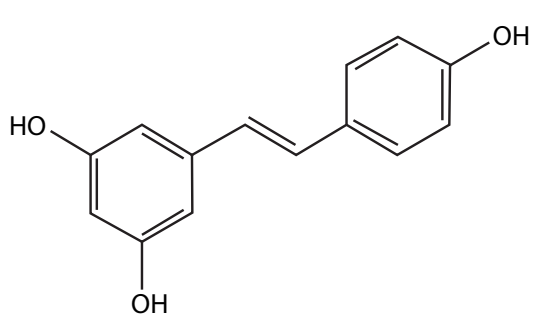

Figure 1: Structure of trans-resveratrol. and expression of hepatic VEGF and HIF- $1 \alpha$ during DENA-initiated rat liver carcinogenesis. The liver tissues were harvested from a previously reported chemopreventive study in which rats exposed to dietary resveratrol $(50-300 \mathrm{mg} / \mathrm{kg})$ exhibited $22-60 \%$ inhibition of hepatic tumor formation, respectively [29]. In this study, we have demonstrated for the first time that resveratrol remarkably inhibited angiogenesis by suppressing the expression of VEGF and HIF- $1 \alpha$ in the liver subjected to DENA-initiated hepatocellular transformation, which may represent a novel molecular mechanism of resveratrol chemoprevention of liver cancer.

\section{Materials and Methods}

\section{Chemicals}

Trans-resveratrol ( $98 \%$ pure) was purchased from Organic Herb, Inc. (Changsha, Hunan, P.R. China). DENA and PB were obtained from Sigma-Aldrich (St. Louis, MO). Goat polyclonal platelet/endothelial cell adhesion molecule-1 (PECAM-1, also referred to as CD31) antibody, mouse polyclonal VEGF antibody, rabbit polyclonal HIF-1 $\alpha$ antibody and $\mathrm{ABC}$ staining systems were purchased from Santa Cruz Biotechnology (Santa Cruz, CA). All other chemicals and reagents were procured from Fisher Scientific (Pittsburgh, PA).

\section{Animals and treatment}

Liver samples utilized in the present study were originated from our previously reported chemopreventive study following our established two-stage rat hepatocarcinogenesis protocol [29]. All animal procedures were approved by the Institutional Animal Care and Use Committee at the Northeastern Ohio Universities Colleges of Medicine and Pharmacy in accordance with National Institutes of Health guidelines. Briefly, pathogen-free female Sprague-Dawley rats (Charles River Laboratories, Wilmington, MA), were divided into five groups. While one group served as the vehicle control (normal group), initiation of hepatocarcinogenesis in all other four groups was performed by a single intraperitoneal injection of DENA at a dose of $200 \mathrm{mg} / \mathrm{kg}$, followed by promotion with PB at a concentration of $0.05 \%(\mathrm{w} / \mathrm{v})$ in drinking water, which was started two weeks after the DENA injection and continued until the end of the study. Rats had free access either to a pulverized standard diet (for normal and DENA control groups) or the same diet supplemented with various amounts of resveratrol equivalent to a daily dose of 50,100 or $300 \mathrm{mg} / \mathrm{kg}$ body weight (for the three resveratrol-treated groups). Treatment with resveratrol was initiated 4 weeks prior to DENA injection and continued for 20 consecutive weeks. At the end of the study (20 weeks), the livers from several groups were subjected to perfusion and subsequently excision under anesthesia. The results on the incidence, multiplicity and size distribution of hepatic nodules have already been reported [29]. A portion of the non-nodular liver tissue from various rat groups was collected, snap-frozen in liquid nitrogen and subsequently stored in a freezer at $-80^{\circ} \mathrm{C}$. These liver tissues were utilized for various assays described below. The rationale for using non-nodular tissue is the fact that DENA produced nodules of different sizes which may cause different hypoxic conditions resulting in differential induction of VEGF [49]. This could introduce errors in the interpretation and comparison of experimental results.

\section{Immunohistochemical analysis}

Immunohistochemical detection of CD31, VEGF and HIF-1 $\alpha$ in $\sim 10-\mu \mathrm{m}$ thick hepatic sections was performed by standard immunohistochemical techniques. In short, the sections were hydrated in $1 \mathrm{X}$ phosphate-buffered saline (PBS) for $5 \mathrm{~min}$. Antigen retrieval was performed by incubating the sections in $10 \mathrm{mM}$ sodium 
citrate buffer $(\mathrm{pH} 6.0)$ at $80^{\circ} \mathrm{C}$ for $10 \mathrm{~min}$. The sections were cooled to room temperature for $20 \mathrm{~min}$. All ensuing steps were carried out at room temperature. Following a 5-min wash with $1 \mathrm{X}$ PBS, the endogenous peroxidases were blocked by $1 \%$ hydrogen peroxide in PBS for $5 \mathrm{~min}$. The sections were washed as before and blocked for $1 \mathrm{~h}$ in PBS containing 1.5\% normal serum. The slides were then incubated overnight with primary antibodies against CD31, VEGF and HIF-1 $\alpha$ (1:50 dilution) at $4^{\circ} \mathrm{C}$ in a humidified chamber. After washing with PBS, the sections were incubated with horseradish peroxidase (HRP)-conjugated secondary antibodies at 1:100 dilutions for 30 min at $37^{\circ} \mathrm{C}$. The immune reactions were visualized by immersing the slides in 3,3'-diaminobenzidine tetra hydrochloride reagent. The sections were counterstained with hematoxylin. Negative control sections were processed simultaneously with the omission of the primary antibodies. All sections were dehydrated, mounted with cover slips and viewed under a light microscope (VisaVision, VWR International, West Chester, PA).

\section{Determination of angiogenesis}

Hepatic microvessel density (MVD) was used as an index of angiogenesis. The MVD was measured by immunohistochemical staining of liver specimens with antibody specific for CD31 expression by vascular endothelial cells. The MVD was determined following the procedure of Weinder et al. [50] with modifications. In short, two to three systematically spread areas of high-density microvessels (defined as any brown-stained endothelial cell or cellular cluster clearly separated from adjacent blood vessels, tumor cells and connective tissue elements) per sample were selected and the number of CD31positive brown microvessels was counted. Neither the presence of red blood cells nor a vessel lumen was required for a structure to be considered as a microvessel. The results were expressed as the mean number of vessels per high power $(100 \times)$ field. The mean MVD quantity was calculated based on 4 random fields per animal and 4 animals per group.

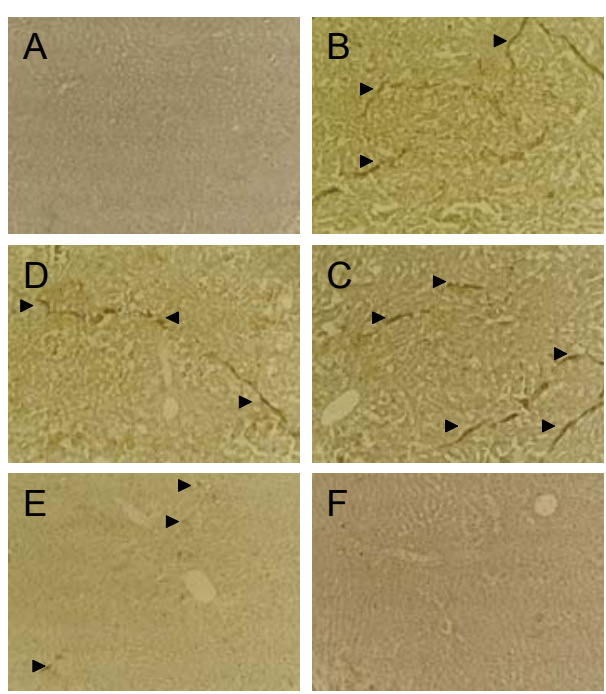

Figure 2: Effects of resveratrol on hepatic CD31 expression during DENA-initiated hepatocarcinogenesis in female Sprague-Dawley rats. Rats were sacrificed 20 weeks following the commencement of the study and immunohistochemistry was performed to detect CD31. Arrowheads indicate immunohistochemical staining of CD31 (magnification: 100x). (A) Near absence of immunopositivity in normal liver; $(B)$ and $(C)$ intense immunoreactivity in DENA control liver; (D) no alteration of expression in $50 \mathrm{mg} / \mathrm{kg}$ resveratrol group; (E) decreased expression in $100 \mathrm{mg} / \mathrm{kg}$ resveratrol group; and $(\mathrm{F})$ near absence of CD31 expression in $300 \mathrm{mg} / \mathrm{kg}$ resveratrol group are noticed.

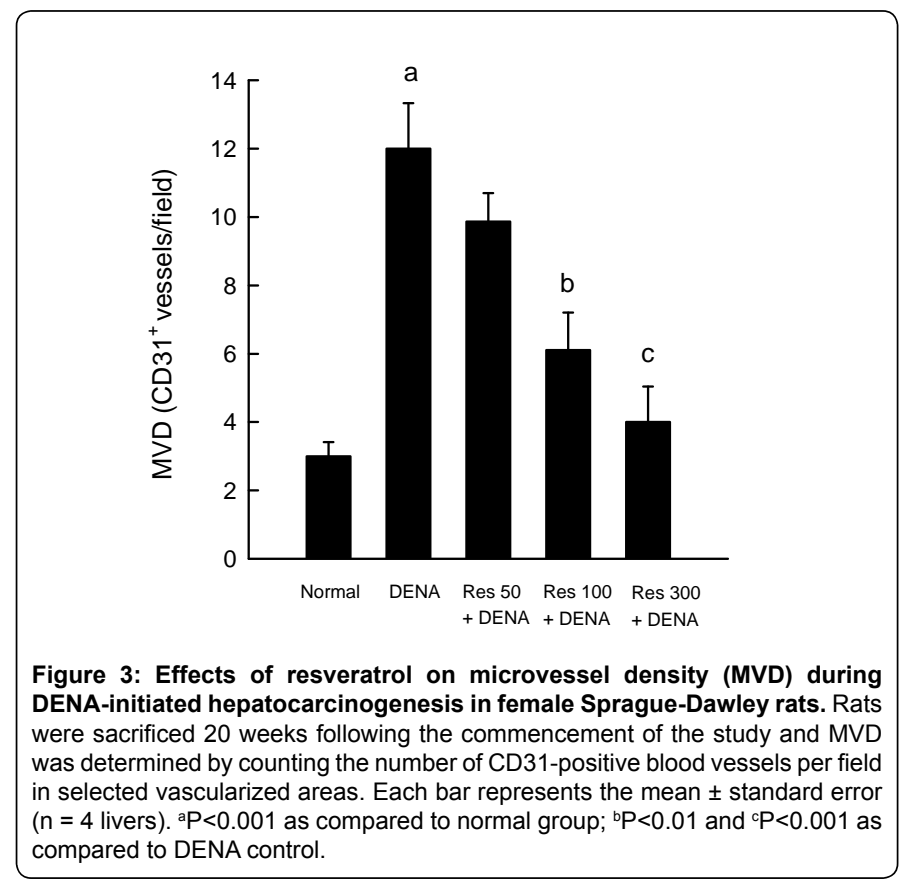

\section{Expression of VEGF and HIF-1 $\alpha$}

Immunohistochemically-positive cells for VEGF and HIF-1 $\alpha$ showed brown granules predominantly in the cytoplasm and nucleus, respectively. One thousand cells from at least 4 separate tissue sections were analyzed per animal and 4 animals per group were used for immunohistochemical analysis. The percentage of positive cells was calculated. The expression of VEGF and HIF-1 $\alpha$ were evaluated semi-quantitatively on the basis of the percentage of immunopositive cells.

\section{Statistical analysis}

Results are presented as mean \pm SEM. One-way analysis of variance was performed to estimate overall significance followed by post-hoc analysis using the Student-Neuman-Keuls test. A P-value less than 0.05 was considered to be statistically significant. All statistical analyses and graphical representation were performed using a commercial software program (SigmaStat 3.1, Systat Software, Inc., San Jose, CA).

\section{Results}

\section{Resveratrol inhibits angiogenesis in DENA-induced rat liver carcinogenesis}

Angiogenesis is not only involved in the growth and progression of liver tumor, but it also plays a crucial role in early events of multistage hepatocarcinogenesis $[18,19]$. Accordingly, we investigated the antiangiogenic effects of resveratrol during DENAinitiated hepatocarcinogenesis in rats by immunostaining of hepatic sections for an endothelial cell marker, CD31. While CD31-positive cells were seldom found in normal rat livers (Figure 2A), there was an increase in the expression of immunopositive cells in DENA-treated rats as compared to normal rats (Figures $2 \mathrm{~B}$ and $2 \mathrm{C}$ ), indicating elevated angiogenesis. Treatment with resveratrol at $50 \mathrm{mg} / \mathrm{kg}$ did not alter the expression of CD31-positive cells in DENA-treated animals (Figure 2D). However, there was a decrease in CD31 expression in the livers of rats treated with resveratrol with a dose of 100 (Figure 2E) or $300 \mathrm{mg} / \mathrm{kg}$ (Figure 2F), with the most significant decrease 
occurring in the latter group. We have also used CD31 as a marker for MVD estimation, and MVD values have been compared among the various animal groups (Figure 3). DENA treatment alone afforded a significant $(\mathrm{P}<0.001)$ increment in MVD compared to normal. Although resveratrol treatment with $50 \mathrm{mg} / \mathrm{kg}$ did not influence the MVD compared to DENA control, a statistically significant $(\mathrm{P}<0.01$ or 0.001 ) decrease in MVD value was noticed following the medium $(100 \mathrm{mg} / \mathrm{kg})$ or high $(300 \mathrm{mg} / \mathrm{kg})$ dose treatment group of resveratrol, respectively (Figure 3). Our data indicate that resveratrol suppresses DENA-evoked angiogenesis in rat liver in a dose-dependent fashion.

\section{Resveratrol suppresses VEGF expression during DENA-initiated hepatocarcinogenesis}

As VEGF is involved in the angiogenic process, we next examined whether or not the inhibitory effect of resveratrol on DENAstimulated angiogenesis was correlated with VEGF expression. There was a significant increase in VEGF immunostaining in the cytoplasm of cells from DENA control animals (Figures $4 B$ and $4 C$ ) as compared to normal counterparts (Figures 4A). A marginal change in the expression of VEGF following the low dose of resveratrol $(50 \mathrm{mg} / \mathrm{kg}$, Figure 4D) and significant reduction in VEGF expression by medium $(100 \mathrm{mg} / \mathrm{kg}$, Figure $4 \mathrm{E})$ or high $(300 \mathrm{mg} / \mathrm{kg}$, Figure $4 \mathrm{~F})$ dose of resveratrol were noticed. The semi-quantitative evaluation of VEGF expression in several groups is depicted in Figure 5. DENA elicited more than an 18 -fold increase $(\mathrm{P}<0.001)$ in VEGF expression as compared to normal animals. There was no difference between the VEGF expression of DENA control group and resveratrol $(50 \mathrm{mg} / \mathrm{kg})$ plus DENA group. We also found that VEGF expression was markedly $(\mathrm{P}<0.001)$ suppressed by resveratrol at $100 \mathrm{mg} / \mathrm{kg}$ or $300 \mathrm{mg} / \mathrm{kg}$ in the liver of DENA-exposed rats, and the extent of this inhibitory effect was similar in magnitude to the suppression of neovascularization as measured by MVD.
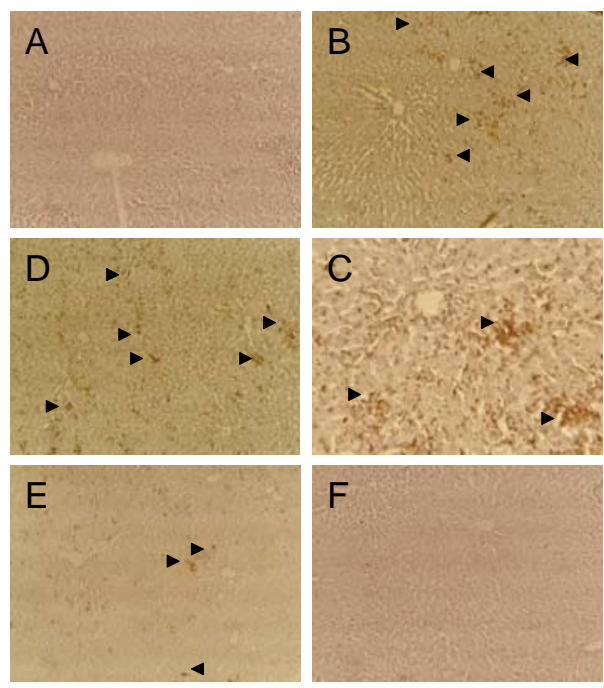

Figure 4: : Effects of resveratrol on hepatic vascular endothelial growth factor (VEGF) expression during DENA-initiated hepatocarcinogenesis in female Sprague-Dawley rats. Rats were sacrificed 20 weeks following the commencement of the study and immunohistochemistry was performed to detect VEGF. Arrowheads indicate immunohistochemical staining of VEGF. (A) Near absence of immunopositivity in normal liver (100); (B) and (C) intense immunoreactivity in DENA control liver (100 and 250x respectively); (D) no alteration of expression in $50 \mathrm{mg} / \mathrm{kg}$ resveratrol group (100x); (E) decreased expression in $100 \mathrm{mg} / \mathrm{kg}$ resveratrol group (100x); and $(F)$ near absence of VEGF expression in $300 \mathrm{mg} / \mathrm{kg}$ resveratrol group (100x) are noticed.
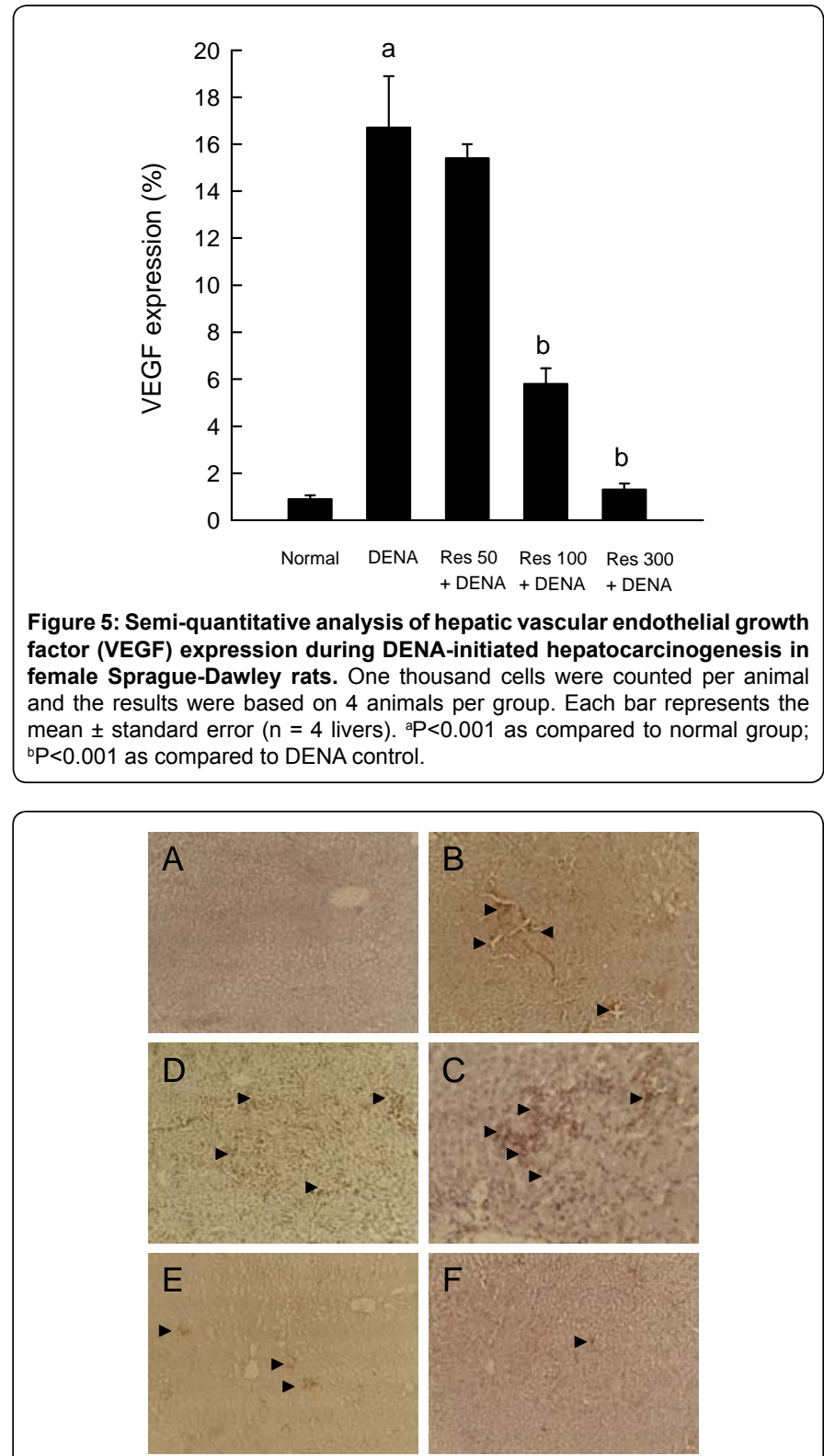

Figure 6: Effects of resveratrol on hepatic hypoxia inducible factor (HIF)-1a expression during DENA-initiated hepatocarcinogenesis in female Sprague-Dawley rats. Rats were sacrificed 20 weeks following the commencement of the study and immunohistochemistry was performed to detect HIF-1a. Arrowheads indicate immunohistochemical staining of HIF-1a. (A) Near absence of immunopositivity in normal liver (100x); (B) and (C) intense immunoreactivity in DENA control liver (100 and 250x respectively); (D) no alteration of expression in $50 \mathrm{mg} / \mathrm{kg}$ group (100x); (E) decreased expression in $100 \mathrm{mg} / \mathrm{kg}$ resveratrol group (100x); and (F) near absence of HIF-1 $\alpha$ expression in $300 \mathrm{mg} / \mathrm{kg}$ resveratrol group $(100 \times)$ are noticed.

\section{Resveratrol suppresses HIF-1 $\alpha$ expression during DENA hepatocarcinogenesis}

Since the transcription factor HIF-1 $\alpha$ regulates the expression of VEGF, we further investigated whether or not resveratrol-mediated suppression of VEGF expression was linked to the inhibition of HIF$1 \alpha$. Our immunohistochemical results reveal almost no expression of HIF- $1 \alpha$ in the livers of normal animals (Figure 6A). On the other hand, a substantial increase in HIF-1 $\alpha$ immunoreactivity was noticed in the cell nucleus in liver sections generated from DENA-initiated animals 


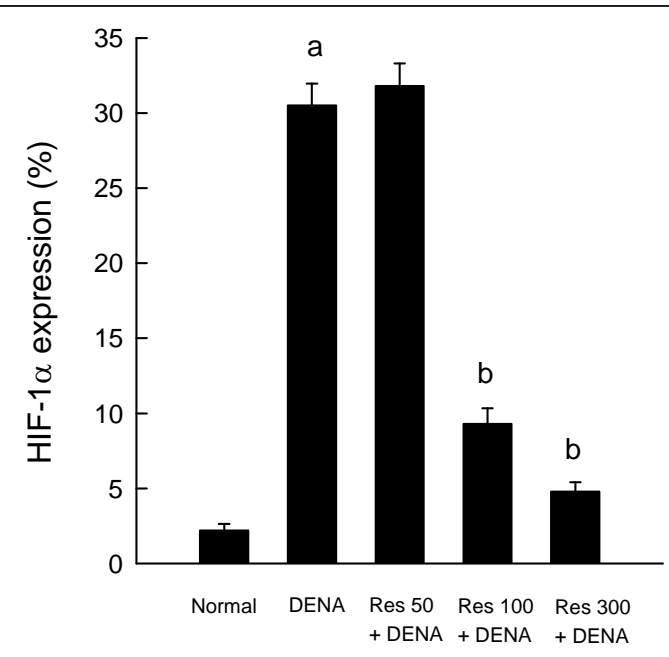

Figure 7: Semi-quantitative analysis of hepatic hypoxia inducible factor (HIF)-1a expression during DENA-initiated hepatocarcinogenesis in female Sprague-Dawley rats. One thousand cells were counted per animal and the results were based on 4 animals per group. Each bar represents the mean \pm standard error ( $n=4$ livers). ${ }^{a} P<0.001$ as compared to normal group; ${ }^{\mathrm{b}} \mathrm{P}<0.001$ as compared to DENA control.

(Figures 6B and C). While resveratrol at $50 \mathrm{mg} / \mathrm{kg}$ did not modify HIF$1 \alpha$ expression (Figure 6D), a moderate reduction of immunoreactivity by $100 \mathrm{mg} / \mathrm{kg}$ (Figure 6E) and a maximum inhibition by $300 \mathrm{mg} / \mathrm{kg}$ (Figure 6F) resveratrol were observed. Figure 7 shows the percentage of hepatic HIF-1-positive cells in various groups of animals. There was a significant $(\mathrm{P}<0.001)$ increase in HIF-1 $\alpha$-positive cells in DENA control group compared to normal control. Resveratrol treatment at a dose of $100 \mathrm{mg} / \mathrm{kg}$ or $300 \mathrm{mg} / \mathrm{kg}$ significantly $(\mathrm{P}<0.001)$ attenuated the hepatic HIF-1 $\alpha$-protein expression in animals challenged with DENA compared to DENA control animals.

\section{Discussion}

HCC is one of the most prevalent and lethal cancers and the incidence of this cancer are expected to rise in the United States as well as rest of the world. Considering the limited treatment options and grave prognosis of HCC, preventive control, especially chemoprevention, has been proposed as the best strategy for considerably lowering the prevalence of this disease. Emerging experimental data based on in vitro and in vivo studies provide substantial evidence that a variety of dietary agents have the potential of preventing HCC [51-54]. Resveratrol, a polyphenolic antioxidant and antiinflammatory agent of dietary origin, has shown great promise as a chemopreventive agent against oxidative stress and inflammation-driven HCC $[28,55]$. We have previously reported the novel finding that dietary resveratrol significantly inhibits experimental hepatocarcinogenesis in rats initiated with the environmental hepatocarcinogen DENA by inhibiting cell proliferation and inducing apoptosis [29]. We have also found that DENA-induced rat hepatocarcinogenesis involves generation of oxidative stress and inflammation, and dietary resveratrol ameliorates oxidative stress and suppresses inflammatory cascades in a doseresponsive fashion during DENA-initiated rat liver carcinogenesis $[30,31]$. In the present study, we have shown for the first time that antiangiogenic mechanisms are also involved in resveratrol-mediated chemoprevention of experimental hepatocarcinogenesis.

Angiogenesis, a complex and critical process, is essential for the growth and maintenance of solid tumors, especially HCC which represents a malignancy characterized by distinct hypervascularization $[13,15]$. This has been supported by the fact that a solid tumor cannot grow beyond a few millimeters in size without angiogenesis. It has been considered that angiogenesis increases tumor diameter from several hundred microns to $1 \mathrm{~mm}$ when the tumor mass consists of approximately $10^{5}-10^{6}$ cells [56]. Several studies have shown that angiogenesis not only plays an important role in tumor growth, but also in early events of neoplastic transformation, including hepatocarcinogenesis [18,57,58]. Yoshiji and colleagues [59] found that angiogenesis was increased stepwise during DENA-initiated hepatocarcinogenesis in mice. MVD has been considered as a reliable quantitative index for assessment of angiogenesis with prognostic importance in HCC. MVD can be determined by immunohistochemical staining of vascular endothelial cells with an antibody specific to endothelial cell markers, for example BHN9, CD31, CD34, CD105, FVIII-RAb, von Willebrand factor and Ulex europaeus agglutinin 1, and then counting the number of microvessels per unit area. Though the endothelial expression of CD31, CD34 and BNH9 was not detected in cirrhotic and dysplastic nodules, all HCC specimens were found to be positive for one of these endothelial cell markers [19]. Roncalli and coworkers [60] reported an increased CD31 expression in HCC and high-grade dysplastic nodules as compared to low-grade dysplastic nodules, which has significant implication in the diagnosis of HCC. In the present study, we have determined the extent of MVD by immunohistochemical staining

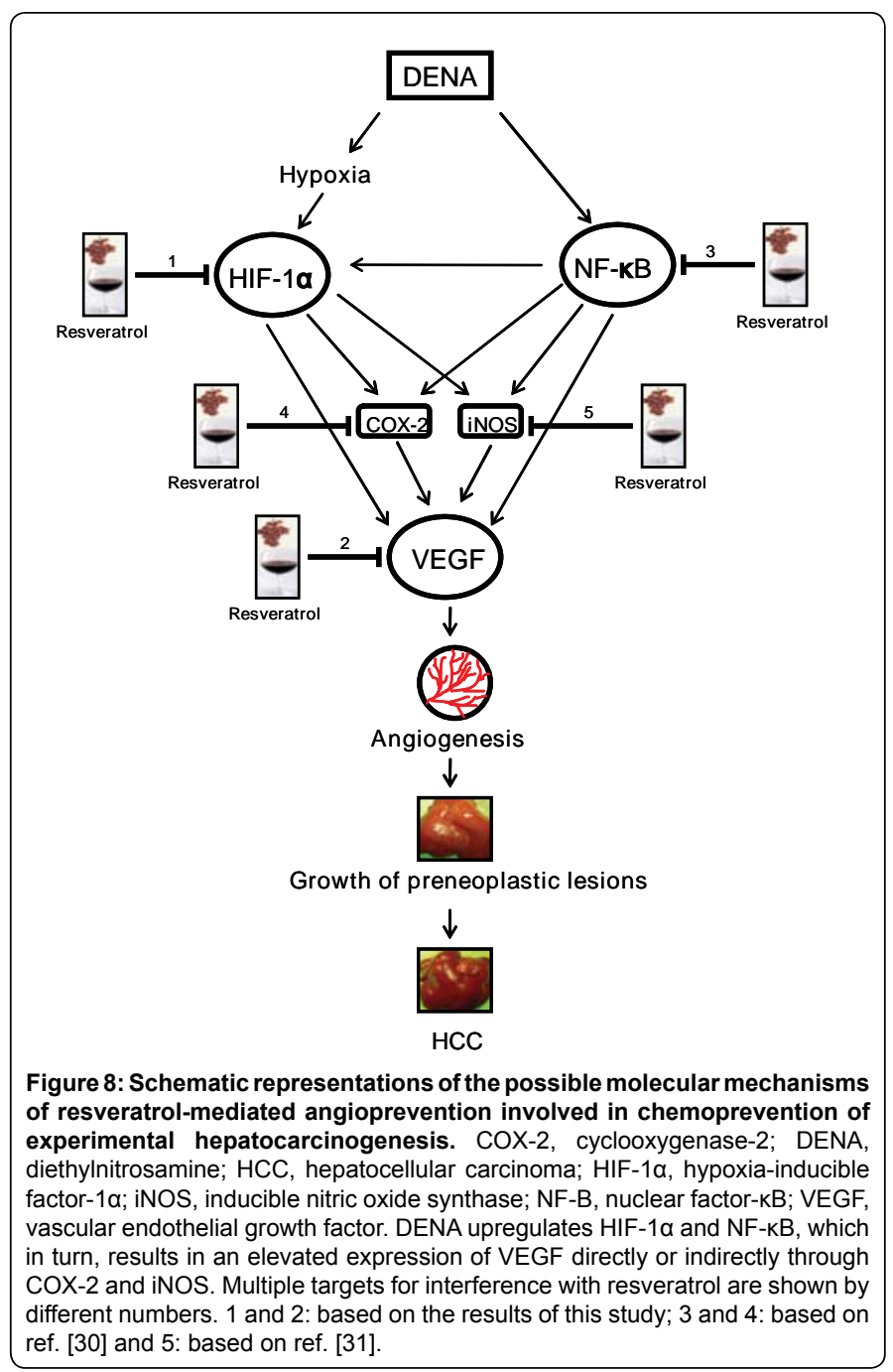


of CD31 cell surface antigen 20 weeks following the administration of the hepatocarcinogen DENA. A strong CD31 staining coupled with a drastic increase in MVD in the liver of DENA-treated animals suggests significant elevation of hepatic neovascularization. A similar increase in CD31 or CD34 immunostaining and MVD during DENA hepatocarcinogenesis in rodents has been previously observed by other groups [59,61-63]. A key observation in this study is the resveratrol-mediated dramatic inhibition of CD31 immunostaining and consequently MVD in hepatic sections of rats exposed to the potent hepatocarcinogen DENA. These results indicate that the suppressive effects on angiogenesis by resveratrol may represent a novel mechanism underlying the chemopreventive activity of this phytochemical against DENA hepatocarcinogenesis. Incidentally, this is the first study showing that attenuation of angiogenesis is involved in resveratrol chemoprevention of hepatic tumorigenesis in vivo. Using the same experimental approach (CD31 immunostaining and MVD), several laboratories have previously shown that reseveratrol attenuates angiogenesis in various tumor cells xenografted in rodents $[36,38,39,43]$

VEGF, also known as the vascular permeability factor, is an endothelial cell-specific mitogen that stimulates endothelial cell proliferation, resulting in the budding of new blood vessels around the growing tumor mass. VEGF mediates its angiogenic effects through several receptors; the two most studied of which are VEGF receptor (VEGFR) 1 (FLT-1 receptor) and VEGFR 2 (KDR/Flk-1) [64]. The VEGF family consists of six different glycoproteins: VEGF-A, VEGF-B, VEGF-C, VEGF-D, VEGF-E and placental growth factor [65]. VEGF-A, commonly referred to as VEGF, is the best characterized VEGF family member. The role of VEGF in HCC-related angiogenesis has been extensively studied, and VEGF seems to be the most important angiogenic factor in HCC [reviewed in ref. 15]. Recent studies have confirmed the correlation between immunohistochemically detected VEGF expression and number of microvessels in HCC [66,67]. Several studies have shown that VEGF also plays a major role in the early stages of hepatocarcinogenesis $[18,59,68]$. Undoubtedly, VEGF signaling is linked to dysregulated angiogenesis in hepatocarcinogenesis, and the disruption of VEGF signaling could have a potential role in blocking angiogenesis during the early events of hepatocellular carcinogenesis. By employing immunohistochemical technique, we have studied the expression of VEGF in DENA-initiated early stage of hepatocarcinogenesis. A striking increase of hepatic VEGF expression in DENA-treated rats provides convincing evidence of elevated VEGF activity that well correlates with elevated angiogenesis, supporting previous studies [62,63]. Resveratrol displayed a conspicuous attenuation of VEGF overexpression in DENA challenged animals, and a near normal level of VEGF expression was achieved with the highest dose. These results clearly suggest that resveratrol is able to abrogate the DENA-induced angiogenic response during hepatocarcinogenesis by downregulating the overexpression of VEGF. The present findings are in agreement with recent reports showing a relationship between resveratrol-mediated inhibition of angiogenesis and a lower VEGF level or expression in chemically-induced cutaneous carcinogenesis [35] or xenografted pancreatic cancer in mice [39].

HIF-1 belongs to the basic helix-loop-helix-periodic acid-Schiff transcription factor family that plays a pivotal role in the angiogenic process. HIF-1 is a heterodimer that consists of two subunits: the oxygen-sensitive HIF- $1 \alpha$ and the constitutively expressed HIF$1 \alpha$ [69]. HIF- $1 \alpha$, the active subunit, is produced in response to hypoxic conditions, where as it is rapidly degraded by the ubiquitinproteosome pathway under normoxic conditions [70]. Under hypoxic conditions, HIF-1 $\alpha$ escapes from degradation, accumulates quickly, subsequently translocates into the nucleus, and heterodimerizes with HIF-1 $\alpha$. The HIF-1 molecule then binds to a cis-acting enhancer element, called "hypoxia-responsive element", and activates transcription of more than 70 survival genes, including VEGF, Flt-1, inducible nitric oxide synthase (iNOS), and cyclooxygenase (COX-2) [71,72]. Additionally, several pathways independent of hypoxia, including inactivation of tumor-suppressor genes as well as stimulation by inflammatory cytokines, homones and growth factors, have been shown to promote the synthesis and stablilization of HIF$1 \alpha$ protein $[73,74]$. Several studies have demonstrated that hypoxiadriven angiogenesis is stimulated via HIF- $1 \alpha$-induced upregulation of VEGF in HCC, underlying the important role of HIF- $1 \alpha$ in angiogenesis and liver tumor growth through regulation of VEGF $[17,75,76]$. Two recent studies have investigated HIF- $1 \alpha$ expression and its association with VEGF expression and angiogenesis during early stages of experimental hepatocarcinogenesis $[62,63]$. Both studies have shown a gradual increase in HIF- $1 \alpha$ expression during the dynamic process of DENA-initiated rat liver carcinogenesis which correlates with elevated VEGF expression and angiogenesis [62,63]. Results of the present investigation also show a dramatic overexpression in HIF$1 \alpha$ in parallel with upregulation of VEGF and increased angiogenesis, indicating the involvement of HIF- $1 \alpha$ in the regulation of VEGFmediated neovascularization during DENA hepatocarcinogenesis. Under these experimental conditions, resveratrol substantially inhibited the expression of HIF- $1 \alpha$, suggesting that this transcription factor is a potential target of resveratrol in the prevention of VEGF-mediated angiogenesis in an experimental model of hepatic neoplasia. Our in vivo data support and strengthen previous in vitro results showing the suppression of HIF-1 and VEGF expression by resveratrol in various tumor cells, including hepatoma cells [4448]. Although the exact mechanisms of resveratrol action on HIF$1 \alpha$ need to be confirmed by further studies, it is highly likely that resveratrol may inhibit HIF-1 $\alpha$ expression via blocking the activation of phosphatidylinositol 3-kinase/Akt and mitogen-activated protein kinase/extracellular signal-related kinase signaling pathways as well as regulation of FOXO transcription factors $[44,46,48,77]$.

In conclusion, the results of the present investigation clearly indicate that DENA-initiated hepatocarcinogenesis in rats exhibits substantial neovascularization possibly due to HIF- $1 \alpha$-mediated overexpression of VEGF. We also provide the first experimental evidence that the dietary polyphenol resveratrol exerts a remarkable angiosuppressive effect during the progression of DENA-evoked hepatocellular carcinogenesis. Resveratrol-mediated inhibition of angiogenesis could be achieved by suppressing VEGF expression through downregulation of HIF-1 $\alpha$. We have previously shown the unique suppressive action of resveratrol against the development of DENA-induced hepatic nodules (precursor of HCC) with a simultaneous inhibition of another transcription factor nuclear factor-kappaB and its downstream target genes, namely COX-2 and iNOS [29-31], which are also the target of HIF- $1 \alpha$. Thus, our current data together with earlier findings suggest that resveratrol exerts potent antiangiogenic activities during the early stage of experimental hepatocarcinogenesis possibly through multiple interactions with the angiogenic cascade (Figure 8). As persistent angiogenic activity represents a hallmark of one of the earliest events in the transformation of a nomal cell to a neoplastic cell, the resveratrol-mediated angiopreventive response may provide vital clues to the molecular basis for its previously observed chemopreventive activity against DENA-induced rat liver carcinogenesis that mimics human liver cancer. Our present findings, in conjunction with an excellent safely profile of resveratrol, reinforce the use of this natural agent in the prevention and treatment of HCC. 
Citation: Bishayee A, Petit DM, Samtani K (2010)Angioprevention is Implicated in Resveratrol Chemoprevention of Experimental Hepatocarcinogenesis. J Carcinogene Mutagene 1:102. doi:10.4172/2157-2518.1000102

\section{Acknowledgements}

This work was partially supported by a Research Incentive Grant from Ohio Board of Regents, State of Ohio, USA. The authors sincerely thank Altaf $S$ Darvesh, Ph.D., and Roslin Thoppil, B.S., for critical reading of the manuscript with helpful suggestions.

\section{References}

1. Parkin DM, Bray F, Ferlay J, Pisani P (2005) Global cancer statistics, 2002. CA Cancer J Clin 55: 74-108.

2. El-Serag HB, Rudolph KL (2007) Hepatocellular carcinoma: epidemiology and molecular carcinogenesis. Gastroenterology 132: 2557-2576.

3. Jemal A, Siegel A, Xu J, Ward E (2010) Cancer statistics, 2010. CA Cancer J Clin 60: 277-300.

4. Schütte K, Bornschein J, Malfertheiner P (2009) Hepatocellular carcinoma epidemiological trends and risk factors. Dig Dis 27: 80-92.

5. Llovet JM, Burroughs A, Bruix J (2003) Hepatocellular carcinoma. Lancet 362: 1907-1917.

6. Bartsch H, Montesano R (1984) Relevance of nitrosamines to human cancer Carcinogenesis 5: 1381-1393.

7. Kensler TW, Egner PA, Wang JB, Zhu YR, Zhang BC, et al. (2004)Chemoprevention of hepatocellular carcinoma in aflatoxin endemic areas. Gastroenterology 127 S310-S318.

8. Bruix J, Sherman M (2005) Management of hepatocellular carcinoma. Hepatology 42: $1208-1236$

9. Senthil M, Mailey B, Leong L, Chung V, Yen Y, et al. (2010) Liver-directed regional therapy in the multi-disciplinary management of hepatocellular cancer. Curr Cancer Ther Rev 6: 19-25.

10. Llovet JM, Ricci S, Mazzaferro V, Hilgard P, Gane E, et al. (2008) Sorafenib in advanced hepatocellular carcinoma. N Eng J Med 359: 378-390.

11. Lu SC (2010) Where are we in the chemoprevention of hepatocellular carcinoma? Hepatology 51: 734-736.

12. Je Y, Schutz FA, Choueiri TK (2009) Risk of bleeding with vascular endothelial growth factor receptor tyrosine-kinase inhibitors sunitinib and sorafenib: a systematic review and mata-analysis of clinical trials. Lancet Oncol 10: 967-974.

13. Sun HC, Tang ZY (2004) Angiogenesis in hepatocellular carcinoma: the retrospectives and perspectives. J Cancer Res Clin Oncol 130: 307-319.

14. Pang R, Poon RTP (2006) Angiogenesis and antiangiogenic therapy in hepatocellular carcinoma. Cancer Lett 242: 151-167.

15. Tanaka S, Arii S (2006) Current status and perspective of antiangiogenic therapy for cancer: hepatocellular carcinoma. Int J Clin Oncol 11: 82-89.

16. Ferrara N (2002) VEGF and the quest for tumour angiogenesis factors. Nat Rev Cancer 2: 795-803.

17. Yasuda S, Arii S, Mori A, Isobe N, Yang W, et al. (2004) Hexokinase II and VEGF expression in liver tumors: correlation with hypoxia-inducible factor 1 alpha and its significance. J Hepatol 40: 117-123.

18. Park YN, Kim YB, Yang KM, Park C (2000) Increased expression of vascular endothelial growth factor and angiogenesis in the early stage of multistage hepatocarcinogenesis. Arch Pathol Lab Med 124: 1061-1065.

19. Frachon S, Gouysse G, Dumortier J, Couvelard A, Nejjari M, et al. (2001) Endothelial cell marker expression in dysplastic lesions of the liver: an immunohistoichemical study. J Hepatol 34: 850-857.

20. Sharma RA, Harris AL, Dalgleish AG, Steward WP, O'Byrne KJ (2001) Angiogenesis as biomarker and target in cancer chemoprevention. Lancer Oncol 2: $726-732$.

21. Tosetti F, Ferrari N, de Flora S, Albini A (2002) 'Angioprevention': angiogenesis is a common and key target for cancer chemopreventive agents. FASEB J 16: 2-14.

22. Cao Y, Cao R, Bråkenheilm E (2002) Antiangiogenic mechanisms of diet-derived polyphenols. J Nutr Biochem 13: 380-390.

23. Bhat TA, Singh RP (2008) Tumor angiogenesis - a potential target in cancer chemoprevention. Food Chem Toxicol 46: 1334-1345.

24. Araldi EMV, Dell'Aica I, Sogno I, Lorusso G, Garbisa S, et al. (2008) Natural and synthetic agents targeting inflammation and angiogenesis for chemoprevention of prostate cancer. Curr Cancer Drug Target 8: 146-155.
25. Bishayee A (2010) Breast cancer prevention and treatment with dietary agents affecting angiogenesis: progress and promise: Angiogenesis and Therapeutic Targets in Cancer. Edited by Chatterjee M, Oak Park: Bentham Science Publisher Ltd., pp. 31-44.

26. Aggarwal BB, Bhardwaj A, Aggarwal RS, Seeram NP, Shishodia S, et al. (2004) Role of resveratrol in prevention and therapy of cancer: preclinical and clinical studies. Anticancer Res 24: 2783-2840.

27. Bishayee $A$ (2009) Cancer prevention and treatment with resveratrol: from rodent studies to clinical trials. Cancer Prev Res 2: 409-418.

28. Bishayee A, Politis T, Darvesh AS (2010) Resveratrol in the chemoprevention and treatment of hepatocellular carcinoma. Cancer Treat Rev 36: 43-53.

29. Bishayee A, Dhir N (2009) Resveratrol-mediated chemoprevention of diethylnitrosamine-initiated hepatocarcinogenesis: inhibition of cell proliferation and induction of apoptosis. Chem Biol Interact 179: 131-144.

30. Bishayee A, Waghray A, Barnes KF, Mbimba T, Bhatia D, et al. (2010) Suppression of the inflammatory cascade is implicated in resveratrol chemoprevention of experimental hepatocarcinogenesis. Pharm Res 27: 1080-1091.

31. Bishayee A, Barnes KF, Bhatia D, Darvesh AS, Carroll RT (2010) Resveratro suppresses oxidative stress and inflammatory response in diethylnitrosamineinitiated rat hepatocarcinogenesis. Cancer Prev Res (Phila) 3: 753-763.

32. Mbimba T, Awale P, Bhatia D, Geldenhuys WJ, Darvesh AS, et al. (2010) Alteration of hepatic proinflammatory cytokines is involved in the resveratrolmediated chemoprevention of chemically-induced hepatocarcinogenesis. Curr Pharm Biotechnol, in press

33. Szekanecz Z, Koch AE (2004) Vascular endothelium and immune responses: implications for inflammation and angiogenesis. Rheum Dis Clin North Am 30: 97-114.

34. Kerbel RS (2008) Tumor angiogenesis. N Eng J Med 358: 2039-2049.

35. Yusuf N, Nasti TH, Meleth S, Elmets CA (2009) Resveratrol enhances cellmediated immune response to DMBA through TLR4 and prevents DMBA induced cataneous carcinogenesis. Mol Carcinog 48: 713-723.

36. Belleri M, Ribatti D, Savio M, Stivala LA, Forti L, et al. (2008) $\alpha_{v} \beta_{3}$ Integrindependent antiangiogenic activity of resveratrol stereoisomers. Mol Cancer Ther 7: $3761-3770$.

37. Garvin S, Öllinger K, Dabrosin C (2006) Resveratrol induces apoptosis and inhibits angiogenesis in human breast cancer xenografts in vivo. Cancer Lett 231 113-122.

38. Kimura Y, Sumiyoshi M, Baba K (2008) Antitumor activities of synthetic and natural stilbenes through antiangiogenic action. Cancer Sci 99: 2083-2096.

39. Harikumar KB, Kunnumakkara AB, Sethi G, Diagaradjane P, Anand P, et al. (2010) Resveratrol, a multitargeted agent, can enhance antitumor activity of gemcitabine in vitro and in orthotopic mouse model of human pancreatic cancer. Int $\mathrm{J}$ Cancer 127: 257-268.

40. Lee EO, Lee HJ, Hwang HS, Ahn KS, Chae C et al. (2006) Potent inhibition of Lewis lung cancer growth by heyneanol A from the roots of Vitis amurensis through apoptotic and anti-angiogenic activities. Carcinogenesis 27: 2059-2069.

41. Kimura Y, Okuda H (2001) Resveratrol isolated from Polygonum cuspidatum root prevents tumor growth and metastasis to lung and tumor-induced neovascularization in Lewis lung carcinoma-bearing mice. J Nutr 131: 1844-1849.

42. Tseng SH, Lin SM, Chen JC, Su YH, Huang HY, et al. (2004) Resveratrol suppresses the angiogenesis and tumor growth of gliomas in rats. Clin Cancer Res 10: 2190-2202.

43. Chen JC, Chen Y, Lin JH, Wu JM, Tseng SH (2006) Resveratrol suppresses angiogenesis in gliomas: evaluation by color Doppler ultrasound. Anticancer Res 26: $1237-1245$.

44. Tang X, Zhang Q, Nishitani J, Brown J, Shi S, et al. (2007) Overexpression of human papillomavirus type 16 oncoproteins enhances hypoxia-inducible factor $1 \alpha$ protein accumulation and vascular endothelial growth factor expression in human cervical carcinoma cells. Clin Cancer Res 13: 2568-2576.

45. Park SY, Jeong KJ, Lee J, Yoon DS, Choi WS, et al. (2007) Hypoxia enhances LPA-induced HIF-1 $\alpha$ and VEGF expression: their inhibition by resveratrol. Cancer Lett 258: 63-69.

46. Cao Z, Fang J, Xia C, Shi X, Jiang BH (2004) trans-3,4,5'-Trihydroxystibene inhibits hypoxia-inducible factor 1 alpha and vascular endothelial growth factor expression in human ovarian cancer cells. Clin Cancer Res 10: 5253-5263. 
Citation: Bishayee A, Petit DM, Samtani K (2010)Angioprevention is Implicated in Resveratrol Chemoprevention of Experimental Hepatocarcinogenesis. J Carcinogene Mutagene 1:102. doi:10.4172/2157-2518.1000102

47. Wu H, Liang X, Fang Y, Qin X, Zhang Y, Liu J (2008) Resveratrol inhibits hypoxiainduced metastasis potential enhancement by restricting hypoxia-induced factor$1 \alpha$ expression in colon carcinoma cells. Biomed Pharmacother 62: 613-621.

48. Zhang Q, Tang X, Lu QY, Zhang ZF, Brown J, et al. (2005) Resveratrol inhibits hypoxia-induced accumulation of hypoxia-inducible factor- $1 \alpha$ and VEGF expression in human tongue squamous cell carcinoma and hepatoma cells. Mol Cancer Ther 4: 1465-1474.

49. Shibuya M (2001) Structure and function of VEGF/VEGF-receptor system involved in angiogenesis. Cell Struct Funct 26: 25-35.

50. Weidner N, Semple JP, Welch WR, Folkman J (1991) Tumor angiogenesis and metastasis - correlation in invasive breast carcinoma. N Eng J Med 324: 1-8.

51. Mann CD, Neal CP, Garcea G, Manson MM, Dennison AR, et al. (2009) Phytochemicals as potential chemopreventive and chemotherapeutic agents in hepatocarcinogenesis. Eur J Cancer Prev 18: 13-25.

52. Glauert HP, Calfee-Mason K, Stemm DN, Tharappel JC, Spear BT (2010) Dietary antioxidants in the prevention of hepatocarcinogenesis: a review. Mol Nutr Food Res 54: 875-896.

53. Bishayee A, Waghray A, Patel MA, Chatterjee M (2010) Vanadium in the detection, prevention and treatment of cancer: the in vivo evidence. Cancer Lett 294: 1-12.

54. Darvesh AS, Bishayee A (2010) Selenium in the prevention and treatment of hepatocellular carcinoma. Anticancer Agents Med Chem 10: 338-345.

55. Bishayee A (2010) Liver cancer prevention and treatment with resveratrol: Liver Cancer: Causes, Diagnosis and Treatment. Edited by Columbus F, Hauppauge: Nova Science Publishers, Inc., in press.

56. Folkman J (1971) Tumor angiogenesis: therapeutic implications. N Eng J Med 285: 1182-1186.

57. Bolontrade MF, Stern MC, Binder RL, Zenklusen JC, Gimenez-Conti IB, et al. (1998) Angiogenesis is an early event in the development of chemically-induced skin tumors. Carcinogenesis 19: 2107-2113.

58. Bergers G, Javaherian K, Lo KM, Folkman J, Hanahan D (1999) Effects of angiogenesis inhibitors on multistage carcinogenesis in mice. Science 284: 808812

59. Yoshiji H, Kuriyama S, Yoshii J, Ikenaka Y, Noguchi R, et al. (2004) Halting the interaction between vascular endothelial growth factor and its receptors attenuates liver carcinogenesis in mice. Hepatology 39: 1517-1524.

60. Roncalli M, Roz E, Coggi G, Di Rocco MG, Bossi P, et al. (1999) The vascular profile of regenerative and dysplastic nodules of the cirrhotic liver: implications for diagnosis and classification. Hepatology 30: 1174-1178.

61. Borbath I, Leclercq IA, Sempoux C, Abarca-Quinones J, Desaeger C, et al. (2010) Efficacy of lanreotide in preventing the occurrence of chemically induced hepatocellular carcinoma in rats. Chem-Biol Interact 183: 238-248.

62. Bozova S, Elpek GO (2007) Hypoxia-inducible factor-1 $\alpha$ expression in experimental cirrhosis: correlation with vascular endothelial growth factor expression and angiogenesis. APMIS 115: 795-801.
63. Wang W, Xu GL, Jia WD, Wang ZH, Li JS, et al. (2009) Expression and correlation of hypoxia-inducible factor- $1 \alpha$, vascular endothelial growth factor and microvessel density in experimental rat hepatocarcinogenesis. J Int Med Res 37: 417-425.

64. Finn RS, Zhu AX (2009) Targeting angiogenesis in hepatocellular carcinoma: focus on VEGF and bevacizumab. Expert Rev Anticancer Ther 9: 503-509.

65. Kaseb AO, Hanbali A, Conant M, Hassan MM, Wollner I, et al. (2009) Vascular endothelial growth factor in the management of hepatocellular carcinoma. Cancer 115: 4895-4906.

66. Brodsky SV, Mendelev N, Melamed M, Ramaswamy G (2007) Vascular density and VEGF expression in hepatic lesions. J Gastrointestin Liver Dis 16: 373-377.

67. Tseng PL, Tai MH, Huang CC, Wang CC, Lin JW, et al. (2008) Overexpression of VEGF is associated with positive p53 immunostaining in hepatocellular carcinoma (HCC) and adverse outcome of HCC patients. J Surg Oncol 98: 349-357.

68. Lee SW, Lee YM, Bae SK, Murakami S, Yun Y, et al. (2000) Human hepatitis $B$ virus $X$ protein is a possible mediator of hypoxia-induced angiogenesis in hepatocarcinogenesis. Biochem Biophys Res Commun 268: 456-461.

69. Wang GL, Jiang BH, Rue EA, Semenza GL (1995) Hypoxia-inducible factor 1 is a basic-helix-loop-helix-PAS heterodimer regulated by cellular $\mathrm{O} 2$ tension. Proc Natl Acad Sci USA 92: 5510-5514.

70. Salceda S, Caro J (1997) Hypoxia-inducible factor 1alpha (HIF-1alpha) protein is rapidly degraded by the ubiquitin-proteosome system under normoxic conditions. Its stabilization by hypoxia depends on redox-induced changes. J Biol Chem 272 22642-22647.

71. Harris AL (2002) Hypoxia - a key regulator in angiogenesis. Nat Rev Cancer 2 38-47.

72. Hirota K, Semenza GL (2006) Regulation of angiogenesis by hypoxia-inducible factor 1. Crit Rev Oncol Hematol 59: 15-26.

73. Kamura T, Sato S, Iwai K, Czyzyk-Krzeska M, Conaway RC, et al. (2000) Activation of HIF1alpha ubiquitination by a reconstituted von Hippel-Lindau (VHL) tumor suppressor complex. Proc Natl Acad Sci USA 97: 10430-10435.

74. Sandau KB, Zhou J, Kietzmann T, Brüne B (2001) Regulation of the hypoxiainducible factor 1 alpha by the inflammatory mediators nitric oxide and tumor necrosis factor-alpha in contrast to desferroxamine and phenylarsine oxide. J Biol Chem 276: 39805-38911.

75. Yang ZF, Poon RT, To J, Ho DW, Fan ST (2004) The potential role of hypoxia inducible factor $1 \alpha$ in tumor progression after hypoxia and chemotherapy in hepatocellular carcinoma. Cancer Res 64: 5496-5503.

76. Huang G, Yang LY, Lu WQ (2005) Expression of hypoxia-inducible factor $1 \alpha$ and vascular endothelial growth factor in hepatocellular carcinoma: impact on neovascularization and survival. World J Gastroenterol 11: 1705-1708.

77. Srivastava RK, Unterman TG, Shankar S (2010) FOXO transcription factors and VEGF neutralizing antibody enhance antiangiogenic effects of resveratrol. Mol Cell Biochem 337: 201-212. 